\title{
OData protocol for Infinispan
}

\author{
Sarith Divakar M \\ Assistant Professor, Computer Science, Cochin College of Engineering and Technology, Valanchery, India
}

\begin{abstract}
Modern application demands high throughput and scalability. Achieving high level of throughput and scalability requires deploying applications in computer grids. But this results in intensifying database bottlenecks. Infinispan is a key/value NoSQL data store and it is a data grid solution written in Java. Infinispan is exceptionally quick even when it deals with a large number of concurrent accesses. Infinispan have an end point called REST endpoint that uses http/web standards for providing services. If you want to connect to Infinispan data grids from non java clients, REST is the solution. Data inserted into the grid is replicated and can be accessed by client application from nearest location. The Open Data Protocol (OData) is an alternative endpoint. It was developed for querying and updating data. OData protocol is built upon technologies like HTTP, Atom Publishing Protocol (AtomPub) and JSON. Accessing Infinispan data store from a wide range of applications is possible using OData protocol. Combining the features of Infinispan and OData provides a distributed cache that is having features like scalability, availability and open access to data.
\end{abstract}

Keywords: Infinispan, OData, NoSQL, Data grid, REST

\section{INTRODUCTION}

Biggest challenges long-faced by today's application are unit growth of knowledge. Quantity of knowledge created and consumed is growing in size. It will increase further when complexity of application increases. Centralized solutions for storing and retrieving data aren't possible for several reasons. First reason is that the inability to scale as and once required. Single purpose of failure hinders handiness of the applications and there for reducing potency. Owing to these shortcomings of centralized systems several like data grids. Data grids act as a middleware which will store massive set of data across distributed applications. Data grids scan and distribute large sets of data across a group of servers act over a network, therefore forming a cluster. Middleware ought to be ready to affect numerous platforms and storage systems. Data grids have to be compelled to be optimized to scale go in environments that manufacture and use large amounts of data. Infinispan is that the resolution that has of these options.

Data grids are highly concurrent distributed data structures. Data grids allow addressing of huge amount of data. Data is persisted in application that can be accessed on a fly. Infinispan is a key/value NoSQL data store and it is a data grid solution written in Java. Infinispan data store can run in distributed mode, thus providing extreme scalability and high availability. Infinispan project aims to provide a data structure that is highly concurrent especially on multi-core architectures. Infinispan is exceptionally quick even when it deals with a large number of concurrent accesses. The Open Data Protocol (OData) was developed for querying and updating data. Accessing Infinispan data store from a wide range of applications is possible using OData protocol.

The OData Protocol is an application-level protocol for interacting with data via Restful web services. The protocol supports the outline of data models and also the editing and querying of knowledge consistent with those models. It provides facilities for metadata, a machinereadable description of the data model exposed by a selected data provider.

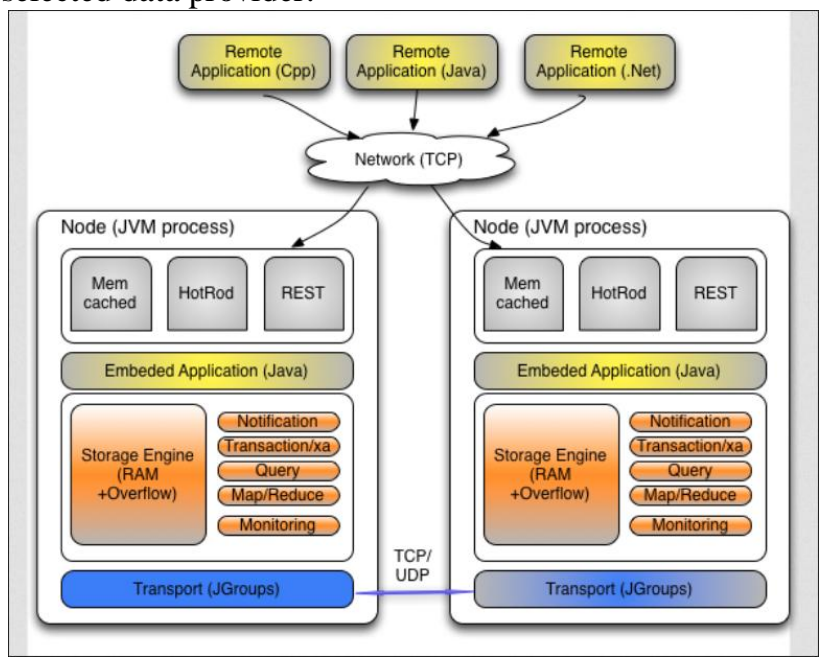

Figure 1.1 Infinispan usage and system architecture [10]

Data means that sets of knowledge entities and also the relationships between them. Querying a data includes requesting that the service perform a group of filtering and alternative transformations to its data then come the results. Editing of data includes creating, editing, and deleting data Operations are performed on data by invoking custom logic. OData allows generic clients to discover the capabilities of services [26]. This is possible because the service is dined in a uniform way, using a common data model. The service advertises its concrete data model in a machine-readable form. An OData service consists of two well-defined, static resources and a set of dynamic resources. The two static resources allow machines to ask a service about its data model. The dynamic resources provide ways to manipulate that model. The metadata document is a static resource that describes the data model and type system understood by that particular OData service. Clients can use the metadata document to 
understand how to query and navigate between the entities in the system. The second static resource in an OData service is the service document. The service document lists all of the top-level entity sets exposed by the service. The rest of an OData service consists of dynamic resources. The URLs for many of these resources can be computed from the information in the metadata document.

\section{ODATA PROTOCOL AND INFINISPAN}

OData protocol is employed to access Infinispan cache. OData protocol provides uniform way to represent data using metadata. It allows computers to know more about the structure of the data. OData provide open methods to query and manipulate data. Advanced queries are also supported to retrieve complex relational data. OData uses JSON or ATOM syntax to describe the payload. GET, POST, PUT, DELETE always have same meaning irrespective of client side applications. In our implemented solution Java user will use prepared OData Cache for putting / getting entries. Infinispan OData server will be capable of understanding an internal structure of stored JSON documents. This hybrid solution will allow clients to query an Infinispan cache using a standardized OData query language, or classic key-value approach.

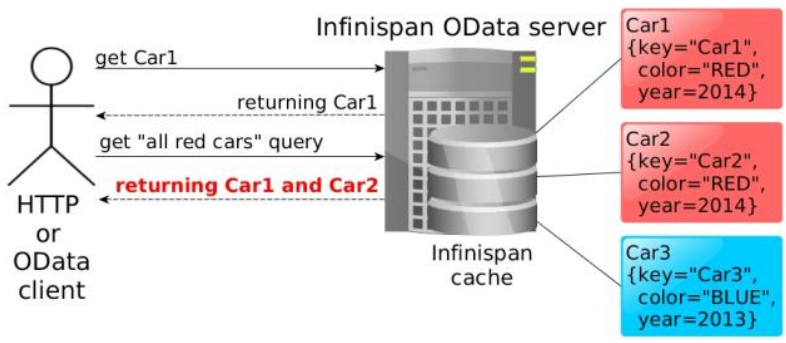

Figure 2.1 Infinispan OData Servers [1]

\section{A. Endpoint Design}

OData endpoint is provided by using Jersey Server. This endpoint starts service (Server Producer) which makes Consumer able to successfully put entries into Infinispan Cache and get entries back from Infinispan Cache via OData protocol. Caches will be configured via starting $\mathrm{xml}$ which will be passed to server as shown in figure 3.1 or programmatically by passing prepared configuration for Cache Manager and Caches. For others, there is a OData Endpoint which reacts and communicates on various type of http requests for putting / getting entries to/from cache. It simply provides interface to in-memory running caches.

\section{B. Infinispan access Design}

Infinispan configuration is passed to set up Cache Manager in memory in OData Jersey Server. It lives in producer. Default access is only to default cache. Otherwise, named caches will be accessible via properly set Entity Set name (=cache name). An operation put/get is called on particular cache in memory of Jersey Server (Producer).

OData protocol suits well for Infinispan key-value NoSQL store. It's a kind of a query language for getting just the entries and the data that we want.

\section{III.QUERYING INFINISPAN}

OData services support querying collections of entities. The target assortment is specified through a URL. Query operations like filter, sort, paging, and projection can be specified as system query. System query options are included in query options module. Infinispan OData server should work as a standalone serve opening up the document store functionality to the vast amount of possible consumers (clients). JSON was decided to be used as an universal format for data contained in documents which will be stored into Infinispan caches. JSON is lightweight, already widely used format and requested by Infinispan users. Additionally, the processing of this format is faster than the processing of XML.

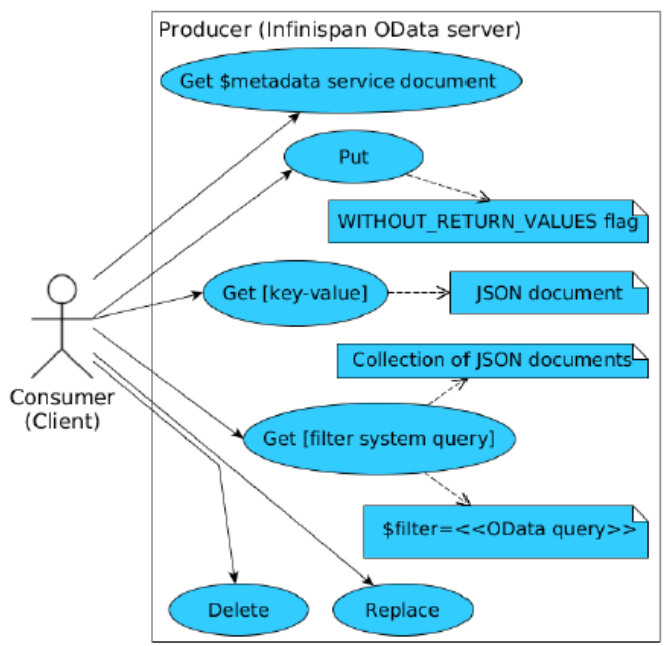

Figure 2.1 Use case UML diagram for Infinispan OData server [1]

\section{IV.CONCLUSION}

Accessing Infinispan data store from a wide range of applications is possible using OData protocol. Combining the features of Infinispan and OData provides a distributed cache that is having features like scalability, availability and open access to data. An alternative REST endpoint similar to Infinispan's existing REST endpoint - that speaks ODATA has been implemented.

\section{ACKNOWLEDGMENT}

I would like to offer my deepest gratitude to everybody who helped me directly and indirectly for the successful completion of my work. I thank God almighty for all the blessings received during this endeavour. Last, but not the least, I thank all my friends and my family for the support and encouragement they have given me during the course of my work.

\section{REFERENCES}

[1] Infinispan OData Server https://github.com/tsykora/infinispanodata-server/

[2] Sarith Divakar M, Ashina Tholiyil. Infinispan as Key / Value data store. International Journal of Advanced Research in Computer and Communication Engineering, Vol. 3, Issue 6, June 2014

[3] SUMATHI, S a S ESAKKIRAJAN. Fundamentals of relational database management systems. London: Springer, 2007, xxv, 776 p. ISBN 35-404-8397-7. 
[4] DATASTAX CORPORATION. Why NoSQL?. In: DataStax [online]. 2013 [cit. 2014-01-04]. Available from: http://www.dat astax.com /wp-content/uploads/2012/10/WPDataStax- WhyNoSQL.pdf

[5] MCCREARY, Dan a Ann KELLY. Making sense of nosql: a guide for managers and the rest of us. S.1.: O'Reilly Media, 2013, 286 p. ISBN 978-161-7291-074.

[6] TIWARI, Shashank C. Professional NoSQL. 1 st ed. Indianapolis, IN: Wiley Publishing, Inc., 2011, p. cm. ISBN 04-709-4224-X.

[7] Getting Started with Infinispan. Infinispan Homepage · Infinispan [online]. 2009-2013 [cit. 2014-01-01]. Available from: http://in finisp an.org/docs/6.0.x/getting_started/getting_started.html

[8] MARKUS, Mircea. What's new in Infinispan 6.0. In: Upload \& Share PowerPoint presentations, documents, infographics [online]. 2013 [cit. 2014-01-01]. Available from: http://www.slideshare. Net /JBUG_London/whats-new-in-infinispan-60/

[9] Infinispan User Guide. Infinispan Homepage · Infinispan [online]. 2009-2013 [cit. 2014-01-01]. Available from: http://infinispan. org/docs/6.0.x/user_guide/user_guide.html

[10] MARKUS, Mircea. What's new in Infinispan 6.0. In: Upload \& Share PowerPoint presentations, documents, infographics [online]. 2013 [cit. 2014-01-01]. Available from: http://www.slideshare. net/JBUG_London/whats-new-in-infinispan-60/

[11] Odata4j - An OData framework for Java [online]. 2013 [cit. 2014 01-04]. Available from: http://www.odata4j.org/

[12] Overview | Open Data Protocol | ODataOpen Data Protocol | OData. Home | Open Data Protocol | ODataOpen Data Protocol | OData [online]. 2013 [cit. 2014-01-04]. Available from: http:// www.odata.org/documentation/overview/ OData Core | Open Data Protocol | ODataOpen Data Protocol | OData. Home | Open Data Protocol | ODataOpen Data Protocol | OData [online]. 2013 [cit. 2014-01-04]. Available from: tp://www. ata .org/documenta tion/odata-v3- documentation/odata-core/

\section{BIOGRAPHY}

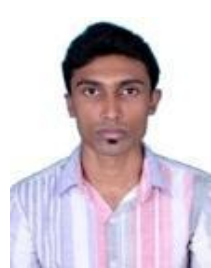

Sarith Divakar $\mathbf{M}$ received his Bachelor's degree in Information Technology in 2009 from Cochin University of Science and Technology, Cochin. In 2014, he earned Master's degree in Computer Science with specialization in Information Systems from Rajagiri School of Engineering and Technology, Cochin Currently working as a faculty member at the Department of Computer Science and Engineering, Cochin College of Engineering \& technology, Valanchery. His research interests include areas like Web services, Data structures, Database systems, NoSQL, Mobile technology. He published various papers in international journals. 